\title{
Determination of whether the association between serum albumin and activities of daily living in frail elderly people is causal
}

\author{
Kaori Kitamura $\cdot$ Kazutoshi Nakamura $\cdot$ \\ Tomoko Nishiwaki $\cdot$ Kimiko Ueno • \\ Akemi Nakazawa $\cdot$ Mariko Hasegawa
}

Received: 4 June 2011/Accepted: 27 July 2011/Published online: 23 August 2011

(C) The Japanese Society for Hygiene 2011

\begin{abstract}
Objectives Serum albumin and activities of daily living (ADL) are associated with each other, but whether the association is causal is not known. The purpose of this study was to determine whether a causal association exists between serum albumin and ADL levels.

Methods The subjects were 116 frail elderly individuals ( 34 men and 82 women; mean age 83.0 years). Demographic characteristics, serum albumin, ADL, and handgrip strength were measured at a baseline examination and at a follow-up examination 2 years later. Levels of ADL were assessed with the Barthel Index. Pearson's correlation coefficients were calculated for serum albumin, ADL, and
\end{abstract}

\footnotetext{
K. Kitamura $\cdot$ T. Nishiwaki

Department of Nursing, Niigata University of Health and Welfare, 1398 Shimami-cho, Kita-ku,

Niigata 951-3198, Japan

K. Nakamura $(\bowtie)$

Department of Community Preventive Medicine,

Niigata University Graduate School of Medical

and Dental Sciences, 1-757 Asahimachi-dori,

Chuo-ku, Niigata 951-8510, Japan

e-mail: kazun@med.niigata-u.ac.jp

K. Ueno

Department of Nursing, Faculty of Medical Technology,

Teikyo University, 2-11-1 Kaga Itabashi-ku,

Tokyo 173-8605, Japan

\section{A. Nakazawa}

Department of Nursing, Tohto College of Health Sciences,

4-2-11 Kamishiba-cho-nishi, Fukaya 366-0052, Japan

M. Hasegawa

Welfare Section, Minamiuonuma City Government,

1188-2 Urasa, Minamiuonuma, Niigata 949-7392, Japan
}

handgrip strength for baseline values and for their 2-year changes $(\Delta)$.

Results At baseline, the mean serum albumin concentration was $4.0 \mathrm{~g} / \mathrm{dL}$ and the total score of the Barthel Index (baseline Barthel Index) was 71.1. The baseline serum albumin level correlated significantly with the baseline Barthel Index $(r=0.287)$ and baseline handgrip strength $(r=0.315)$, but not with $\Delta$ Barthel Index $(r=0.096)$ or $\Delta$ handgrip strength $(r=-0.058)$. The $\Delta$ serum albumin correlated significantly with $\Delta$ Barthel Index $(r=0.296)$, but not with $\Delta$ handgrip strength $(r=0.182)$, baseline Barthel Index ( $r=-0.044)$, or baseline handgrip strength $(r=0.047)$.

Conclusions This 2-year cohort study has demonstrated that a decrease in serum albumin levels is associated with a decrease in ADL levels. A third factor may play a role in adversely affecting both serum albumin and ADL levels in frail elderly people.

Keywords Activities of daily living - Causality · Cohort studies $\cdot$ Frail elderly $\cdot$ Serum albumin

\section{Introduction}

Serum albumin levels and activities of daily living (ADL) are major predictors of mortality in frail elderly people [14]. Epidemiologic studies suggest that serum albumin and ADL are linked [3, 4]. A cohort study has demonstrated that low serum albumin is a risk factor for impaired ADL [3]. In contrast, impaired physical function reduces serum albumin levels [5]. Thus, causality of the association between serum albumin and ADL is unclear. Several recent epidemiologic studies, including cross-sectional $[4,6]$ and cohort $[3,5,7]$ studies conducted in Asian countries, have 
reported an association between serum albumin and ADL levels in elderly people. This has led to increased interest in this topic in Japan. We previously conducted a cohort study assessing mortality risk in a frail Japanese elderly population [4]. In the present study, we evaluated levels of serum albumin and ADL at two time points, which enabled us to explore causality. The aim of this study was to determine whether there was a causal association between serum albumin and ADL in frail elderly people.

\section{Methods}

\section{Subjects}

We targeted 518 elderly people who were approved for care in October 2002, and were living at home in Yamato, Niigata, Japan. Of these, 245 consented to participate in this study. Forty subjects were excluded due to acute illness on the day of the baseline examination. Thus, a total of 205 subjects participated in this study. The baseline study, which included a medical examination and interview, was conducted from January to April 2003. Informed consent was obtained from all subjects and/or their family members before participation in this study. This study was approved by the Ethics Committee of Niigata University School of Medicine. Details of the study procedure have been published previously [8].

\section{Measurements at the baseline examination}

Demographic characteristics, ADL levels, height, weight, handgrip strength, and serum albumin were measured. Levels of ADL were assessed with the Barthel Index [9], which consists of the following 10 activities: feeding, transfers (bed to chair and back), grooming, toilet use, bathing, mobility (on level surfaces), stair climbing, dressing, and bowel and bladder function. Level of assistance required was scored for each item, with 2 to 4 potential scores per item. The total scores range from 0 to 100 , where 0 indicates a high requirement of assistance and 100 indicates a low requirement of assistance. The level of assistance needed for ADL was evaluated by direct interviews. When the subjects themselves could not provide satisfactory answers, people close to them, i.e., staff of the day-service facilities they used, or family members were interviewed. Comorbid diseases were also recorded.

Body height was estimated as being twice the value of the left arm span [10], because height in the standing position could not be measured accurately in many subjects. Body weight was measured with a digital scale (BWB-200S; Tanita, Tokyo, Japan) while the subjects wore light underclothes. Handgrip strength was measured with a digital hand dynamometer (T.K.K.5401; Takei Scientific Instruments, Niigata, Japan) once each for the right and left hands. Blood specimens were collected when the subjects were in a non-fasting state. Serum albumin concentration was determined using the bromcresol green (BCG) method (BML, Tokyo, Japan).

\section{Follow-up examination}

The follow-up examination was conducted 2 years after the baseline examination, in March 2005, in the same setting as the 2003 baseline examination. As in the baseline examination, measurements included ADL levels, height, weight, handgrip strength, and serum albumin. Of the 205 subjects who underwent the baseline examinations, 42 had died and $45 \mathrm{did}$ not participate in the follow-up study. As a result, the present study comprised 118 subjects. Two of these subjects had not undergone blood tests at baseline; therefore, data for a total of 116 subjects (34 men and 82 women) were analyzed.

\section{Statistical analysis}

The 2-year change $(\Delta)$ for a particular variable was calculated by subtracting its baseline value from its follow-up value. Pearson's product-moment correlation coefficients and partial correlation coefficients were calculated, adjusting for sex, age, baseline body mass index (BMI), and the presence or absence of comorbid diseases such as stroke, heart disease, diabetes, hypertension, and respiratory disease, in order to evaluate any associations between two continuous variables. We also conducted multiple logistic regression analysis to assess baseline serum albumin (a predictor) and the $\Delta$ Barthel Index (ADL maintenance or decline as outcomes), adjusting for the possible confounders mentioned above. To make a dichotomous variable for the $\Delta$ Barthel Index, subjects were divided into two classes: a group of subjects for whom ADL was maintained and a group for whom ADL declined during 2 years, with a cutoff of (1) zero or (2) $-1 \mathrm{SD}$ value $(-31.3$ score) of the $\Delta$ Barthel Index. We also analyzed the baseline Barthel Index and $\Delta$ serum albumin was conducted in the same manner $(-1$ SD of $\Delta$ serum albumin, $-0.44 \mathrm{~g} / \mathrm{dL})$. Statistical analyses were performed using the SAS statistical package (release 8.02; SAS Institute, Cary, NC, USA). A $P$ value of $<0.05$ was considered statistically significant.

\section{Results}

The subjects' characteristics at baseline and follow-up, and their changes are shown in Table 1. Mean values for weight, serum albumin, and total score of the Barthel Index 
Table 1 Characteristics [means (standard deviations in parentheses) or proportions] of the 116 subjects at baseline and follow-up, and their respective 2-year changes

\begin{tabular}{lcccc}
\hline & Baseline & Follow-up & 2-Year change & $P$ value \\
\hline Proportion of women $(\%)$ & 70.7 & - & - & - \\
Age (years) & $83.0(8.3)$ & - & - & - \\
Weight $(\mathrm{kg})$ & $47.3(10.1)$ & $46.0(10.2)$ & - & 0.001 \\
Body mass index (BMI; $\left.\mathrm{kg} / \mathrm{m}^{2}\right)$ & $20.6(3.8)$ & - & $-0.11(0.33)$ & - \\
Serum albumin $(\mathrm{g} / \mathrm{dL})$ & $4.0(0.3)$ & $3.9(0.4)$ & $-8.2(23.5)$ & 0.001 \\
Total score of Barthel Index & $71.1(26.0)$ & $62.9(33.7)$ & $0.03(3.58)$ & 0.001 \\
Handgrip strength $(\mathrm{kg})^{\mathrm{a}}$ & $15.6(6.9)$ & $15.8(6.1)$ & 0.941 \\
\hline
\end{tabular}

$\dagger$ Paired $t$ test

a Eight values at baseline and 14 values at follow-up are missing

Table 2 Pearson's correlation coefficients (normal font) and partial correlation coefficients (italics) for baseline Barthel Index (BI), handgrip strength (HS), and serum albumin, and their respective 2-year changes $(\Delta)$

\begin{tabular}{lcccccc}
\hline & Baseline BI & Baseline HS & Baseline albumin & $\Delta$ BI & $\Delta$ HS & $\Delta$ Albumin \\
\hline Baseline BI & - & 0.054 & $0.287^{\dagger}$ & -0.074 & -0.048 \\
Baseline HS & $0.214^{\dagger}$ & - & $0.315^{\dagger}$ & 0.153 & $-0.460^{\dagger}$ & 0.047 \\
Baseline albumin & $0.292^{\dagger}$ & $0.401^{\dagger}$ & - & 0.096 & -0.058 & $-0.263^{\dagger}$ \\
$\Delta$ BI & -0.168 & 0.102 & 0.023 & - & $0.228^{\dagger}$ & $0.296^{\dagger}$ \\
SHS & 0.006 & $-0.545^{\dagger}$ & -0.059 & $0.217^{\dagger}$ & - & 0.182 \\
$\Delta$ Albumin & -0.177 & -0.139 & $-0.360^{\dagger}$ & $0.226^{\dagger}$ & 0.185 & - \\
\hline
\end{tabular}

Partial correlation coefficients (italics) are adjusted for sex, age, baseline BMI, and comorbidities of stroke, heart disease, diabetes, hypertension, and respiratory disease

$\dagger \quad P<0.05$

were significantly different at baseline and follow-up. The subjects' comorbidities were as follows: stroke 25 (21.6\%), heart disease $21(18.1 \%)$, diabetes $8(6.9 \%)$, hypertension $34(29.3 \%)$, and respiratory disease $4(3.5 \%)$. Liver disease was not reported.

Pearson's correlation coefficients and partial correlation coefficients for baseline Barthel Index, handgrip strength, and serum albumin, and their respective 2-year changes $(\Delta)$ are shown in Table 2. The correlation coefficients for baseline serum albumin versus baseline Barthel Index and for baseline serum albumin versus baseline handgrip strength were statistically significant $(P=0.002$ and $P=0.001$, respectively). Baseline serum albumin did not correlate significantly with $\Delta$ Barthel Index or $\Delta$ handgrip strength. Similarly, $\Delta$ serum albumin did not exhibit a significant correlation with baseline Barthel Index or baseline handgrip strength. However, $\Delta$ serum albumin correlated significantly with $\Delta$ Barthel Index (Fig. 1), but not with $\Delta$ handgrip strength. The partial correlation coefficients revealed that these associations held when adjustments were made for possible confounders (Table 2). The partial correlation coefficient between $\Delta$ serum albumin and $\Delta$ Barthel Index was also statistically significant
( $r=0.226, P=0.029$ ), although this was lower than the crude correlation coefficient.

Multiple logistic regression analysis showed that baseline serum albumin did not significantly predict ADL decline when Barthel Index cutoff values of zero (odds ratio $[\mathrm{OR}]=0.59,95 \%$ confidence interval $[\mathrm{CI}]$ 0.19-1.84, $P=0.365)$ or -1 SD (OR $=0.52$, 95\% CI $0.09-3.03$, $P=0.468)$ were used. This analysis also showed that the baseline Barthel Index did not significantly predict serum albumin decline either when using an albumin cutoff value of zero $(\mathrm{OR}=1.01,95 \%$ CI $1.00-1.03, P=0.142)$ or -1 $\mathrm{SD}(\mathrm{OR}=0.99,95 \%$ CI $0.97-1.01, P=0.423)$.

\section{Discussion}

An association between serum albumin and ADL levels has been reported in cross-sectional $[4,11,12]$ and cohort [3, 7] studies. A 12-year cohort study targeting 1844 elderly individuals showed that those with low serum albumin levels had a higher risk of impaired ADL [3]. This suggests that a low serum albumin level may decrease ADL levels. In contrast, another study suggested that impaired ADL 


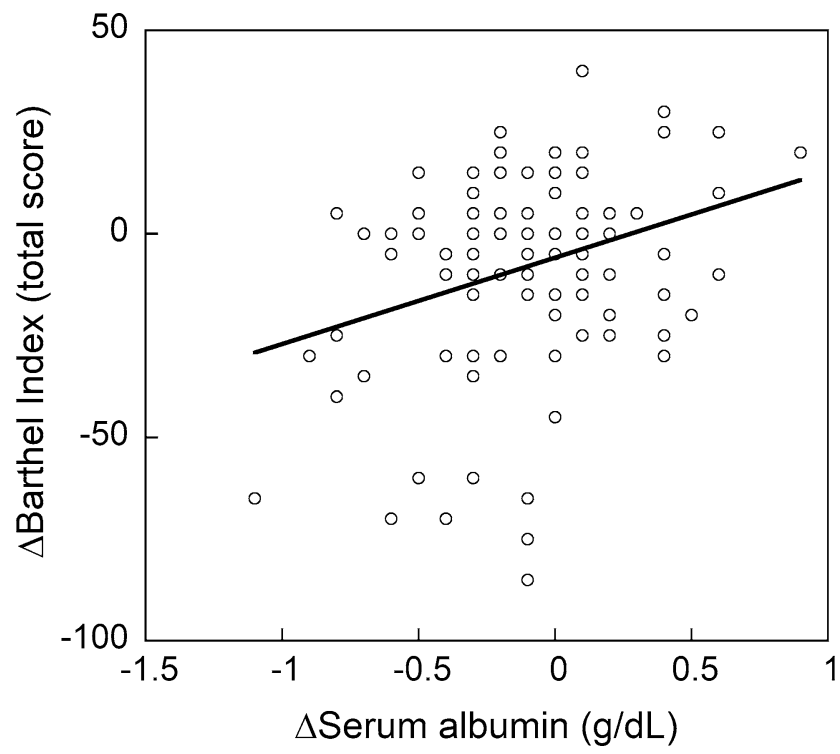

Fig. 1 Correlation between the 2-year changes in serum albumin ( $\Delta$ serum albumin) and 2-year changes in total score of Barthel Index $(\Delta$ Barthel Index $)$. The correlation coefficient is $0.296(P=0.001)$

decreased serum albumin [5]. Physical activity is thought to be involved in the maintenance of serum albumin through an increase in the hepatic synthesis of albumin. In any case, the mechanism underlying this association has not been fully elucidated.

In the present study, we confirmed the close relationship between serum albumin and ADL levels by demonstrating a significant correlation between their 2-year changes. However, baseline serum albumin was not a predictor of a subsequent change in ADL levels, or vice versa. These findings suggest that there is no causality in the association between the two variables. Therefore, a third factor, which affects both serum albumin and ADL levels, may exist. First, decreased dietary intake may be involved in the association. Because the subjects in this study were a frail elderly population, a lower health status could easily cause hypoalbuminemia via low dietary intake and ADL decline. Second, muscle mass, which is associated with both nutritional status and physical disability, is a likely mediator of the association [5]. In our study, however, the 2-year change in handgrip strength did not correlate significantly with the change in serum albumin. This means that muscle mass may not play a role in the association between serum albumin and ADL. Finally, inflammation should be considered, because the serum albumin concentration could be a marker of inflammation. Hypoalbuminemia is thought to be caused by inflammation and inadequate protein and caloric intake in patients with chronic disease [13]. Unfortunately, we did not measure inflammation markers, and, thus, were unable to determine interrelationships between serum albumin, $\mathrm{ADL}$, and inflammation.
The strength of the present study is its prospective cohort design, which is suitable for the detection of causative factors. However, this study also has a number of limitations. The sample size was relatively small, and thus may have failed to detect weak, but significant, associations. A study with a larger sample size is necessary. Furthermore, there were a number of dropouts (42 deaths and 45 non-participants) during the follow-up. Detailed reasons for the non-participants' refusal to participate were unclear, but the majority of them were likely in bad health condition. Considering this, the results of the present study may be generalized to frail elderly people in better health condition, rather than to all frail elderly people.

\section{Conclusions}

The present cohort study has demonstrated that a decrease in the serum albumin level is associated with a decrease in ADL levels, although causality is still unclear. A third factor may play a role in adversely affecting both serum albumin and ADL levels in frail elderly people.

Acknowledgments We wish to thank the subjects for their cooperation in this study. We are also indebted to the staff of Yukiguni Yamato General Hospital and the day-service centers of Yairoen, Nanosatoaiai, Honobono, and Jizonoyu for their help in the organization and data collection for this study. This study was partly supported by a Health and Labour Sciences Research Grant 14-Aging and Health-022, from the Ministry of Health, Labour and Welfare of Japan, and a Grant-in-Aid for Scientific Research (C) No. 14572208, from the Ministry of Education, Culture, Sports, Science and Technology of Japan.

Conflict of interest The authors declare that they have no conflicts of interest.

\section{References}

1. Sahyoun NR, Jacues PF, Dallal G, Russel RM. Use of albumin as a predictor of mortality in community-dwelling and institutionalized elderly populations. J Clin Epidemiol. 1996;49:981-8.

2. Kuzuya M, Masuda Y, Hirakawa Y, Iwata M, Enoki H, Hasegawa $\mathrm{J}$, et al. Day care service use is associated with lower mortality in community-dwelling frail older people. J Am Geriatr Soc. 2006;54:1364-71.

3. Okamura T, Hayakawa T, Hozawa A, Kadowaki T, Murakami Y, Kita Y, et al. Lower levels of serum albumin and total cholesterol associated with decline in activities of daily living and excess mortality in a 12-year cohort study of elderly Japanese. J Am Geriatr Soc. 2008;56:529-35.

4. Kitamura K, Nakamura K, Nishiwaki T, Ueno K, Hasegawa M. Low body mass index and low serum albumin are predictive factors for short-term mortality in elderly Japanese requiring home care. Tohoku J Exp Med. 2010;221:29-34.

5. Kuzuya M, Izawa S, Enoki H, Okada K, Iguchi A. Is serum albumin a good marker for malnutrition in the physically impaired elderly? Clin Nutr. 2007;26:84-90. 
6. Onem Y, Terekeci H, Kucukardali Y, Sahan B, Solmazgül E, Senol MG, et al. Albumin, hemoglobin, body mass index, cognitive and functional performance in elderly persons living in nursing homes. Arch Gerontol Geriatr. 2010;50:56-9.

7. Higashiguchi M, Nakaya N, Ohmori K, Shimazu T, Sone T, Hozawa A, et al. Malnutrition and the risk of long-term care insurance certification or mortality. A cohort study of the Tsurugaya project. Nippon Koshu Eisei Zasshi. 2008;55:433-9. (In Japanese).

8. Nishiwaki T, Nakamura K, Ueno K, Fujino K, Yamamoto M. Health characteristics of elderly Japanese requiring care at home. Tohoku J Exp Med. 2005;205:231-9.

9. Mahoney FI, Barthel DW. Functional evaluation: the Barthel Index. Md State Med J. 1965;14:61-5.
10. Kwok T, Whitelaw MN. The use of armspan in nutritional assessment of the elderly. J Am Geriatr Soc. 1991;39:492-6.

11. Jensen GL, Kita K, Fish J, Heydt D, Frey C. Nutrition risk screening characteristics of rural older persons: relation to functional limitations and health care charges. Am J Clin Nutr. 1997;66:819-28.

12. Romagnoni F, Zuliani G, Bollini C, Leoci V, Soattin L, Dotto S, et al. Disability is associated with malnutrition in institutionalized elderly people. The I.R.A. study. Istituto di Riposo per Anziani. Aging Clin Exp Res. 1999;11:194-9.

13. Don BR, Kaysen G. Serum albumin: relationship to inflammation and nutrition. Semin Dial. 2004;17:432-7. 